\title{
Modeling the Production of Cosmogenic Radionuclides due to Galactic and Solar Cosmic Rays
}

\author{
Konstantin Herbst* and Bernd Heber \\ Christian-Albrechts-Universität zu Kiel, Kiel, Germany \\ E-mail: herbst@physik.uni-kiel.de, heber@physik.uni-kiel.de
}

\section{Jürg Beer}

Swiss Federal Institute of Aquatic Science and Technology, Dübendorf, Switzerland

E-mail: juerg.beer@eawag.ch

\author{
Allan J. Tylka \\ NASA Goddard Space Flight Center, Washington, DC, USA \\ E-mail: allan.j.tylka@nasa.gov
}

\begin{abstract}
Cosmogenic radionuclides such as ${ }^{10} \mathrm{Be},{ }^{14} \mathrm{C}$ and ${ }^{36} \mathrm{Cl}$ are a product of the interaction of high energetic primary particles, in particular galactic cosmic rays (GCR), with the Earth's atmosphere. Because GCRs are modulated on their way through the interplanetary medium the GCR-induced production of these radionuclides is anti-correlated to the solar cycle. Furthermore, during phases of strong solar activity also solar energetic particle (SEP) events occur frequently. While the production due to GCRs can be seen as background production, in particular so-called Ground Level Enhancement (GLE) events, strong SEP events which can be detected at the Earth's surface may strongly contribute to the production of ${ }^{10} \mathrm{Be},{ }^{14} \mathrm{C}$ and ${ }^{36} \mathrm{Cl}$, a topic by now highly discussed in the literature. Using energy spectra of modern GLE events, which have occurred since 1941, we will investigate the influence of 58 out of the 71 GLEs and statistically investigate the possibility to detect such events in present ice-core and tree-ring records.
\end{abstract}

The 34th International Cosmic Ray Conference,

30 July- 6 August, 2015

The Hague, The Netherlands

\footnotetext{
*Speaker.
} 


\section{Introduction}

The main motor for the production of cosmogenic radionuclides such as ${ }^{10} \mathrm{Be},{ }^{14} \mathrm{C}$ and ${ }^{36} \mathrm{Cl}$ are galactic cosmic rays (GCRs), which consist of $\sim 87 \%$ protons, $\sim 12 \%$ alpha particles and $\sim 1 \%$ heavier particles (see e.g. Simpson, 1983). As they enter the heliosphere they are modulated due to the heliospheric magnetic field (see e.g. Scherer et al., 2011; Strauss et al., 2011; Herbst et al., 2012). Thus, during phases of low solar activity much higher particle intensities occur inside the heliosphere then during solar maximum conditions. Because the particle spectrum outside the heliosphere, the so-called local interstellar spectrum (LIS), has not jet been measured in-situ by now multiple LIS models exist in the literature. However, the use of different LIS models has a strong influence on the computation of the cosmogenic radionuclide production rates (see e.g. Herbst et al., 2010). The transport of GCRs in the heliosphere can be described by the Parker equation (Parker, 1965), with which the phase space distribution depending on the main modulation processes such as convection, drifts, diffusion and adiabatic energy changes can be calculated. A widely used, but only first order approximation is the Force-Field approach (see e.g. Caballero-Lopez and Moraal, 2004; Moraal , 2011). Here the transport is only depending on one free parameter, the solar modulation parameter $\phi$. A reconstruction of the modulation parameter values between 1939 and 2010 has been performed e.g. by Usoskin et al. (2011a). Therewith, the time-dependent differential GCR flux at $1 \mathrm{AU}$ can be described by

$$
J(E, \phi)=J_{L I S}(E+\Phi) \frac{(E)\left(E+2 E_{r}\right)}{(E+\Phi)\left(E+\Phi+2 E_{r}\right)},
$$

where $J_{L I S}$ is the differential LIS spectrum, $E_{r}$ the rest energy of the particle and $\Phi$ the solar modulation function which is given by $\Phi=(Z e / A) \phi$, with $Z$ and $A$ as charge and mass number of cosmic ray nuclei.

In addition, particles which arrive at the Earth's vicinity also encounter the terrestrial magnetic field shielding the atmosphere from low energetic primary charged particles and, furthermore, will interact with the atmospheric environment once they enter the terrestrial atmosphere. While lower energetic primary particles mainly lose their energy due to ionization processes in the upper atmosphere (see e.g. Usoskin et al., 2011b) the interaction of high-energetic primary particles deeper inside the atmosphere leads to the evolution of altitude-dependent secondary particle cascades mainly consisting of hadronic particles (see e.g. Simpson, 2000). Of great importance for the production of both short- and long-lived cosmogenic radionuclides thereby in particular are secondary neutrons and protons.

Once cosmogenic radionuclides are produced they are the subject of complex atmospheric mixing and transport mechanisms (see e.g. Heikkilä et al., 2008). After being transported and distributed by atmospheric circulation mechanisms as well as being attached to atmospheric aerosols they are either removed by condensation, like e.g. ${ }^{10} \mathrm{Be}$ and ${ }^{36} \mathrm{Cl}$, or become part of the carbon cycle (see e.g. Dunai, 2010), processes which lead to the storage of the cosmogenic radionuclides in natural archives such as ice sheets, trees or sediments. Here the information about their production and transport of and over thousands of years is preserved. In addition to the GCR component also Solar Energetic Particle (SEP) events may play an important role for the production of cosmogenic radionuclides. These events are characterized by an enhancement of the low energy part of the particle spectrum, enhancements which may last up to severals days (see e.g. Mewaldt et al., 2012). Of special interest for this study are so-called Ground Level Enhancement (GLE) events which show a strong intensity increase up to several GeV (see e.g. Stoker , 1995) and, thus, are able to induce the atmospheric production of secondary particle cascades as well as the production of cosmogenic radionuclides.

Using the Monte-Carlo based simulation code PLANETOCOSMICS (Desorgher, 2006) here we compute the global production rates of ${ }^{10} \mathrm{Be},{ }^{14} \mathrm{C}$ and ${ }^{36} \mathrm{Cl}$ due to GCRs as well as 58 out of the 71 GLE event spectra of the past five solar cycles. The used GLE spectra were reconstructed by a new method analyzing data from 

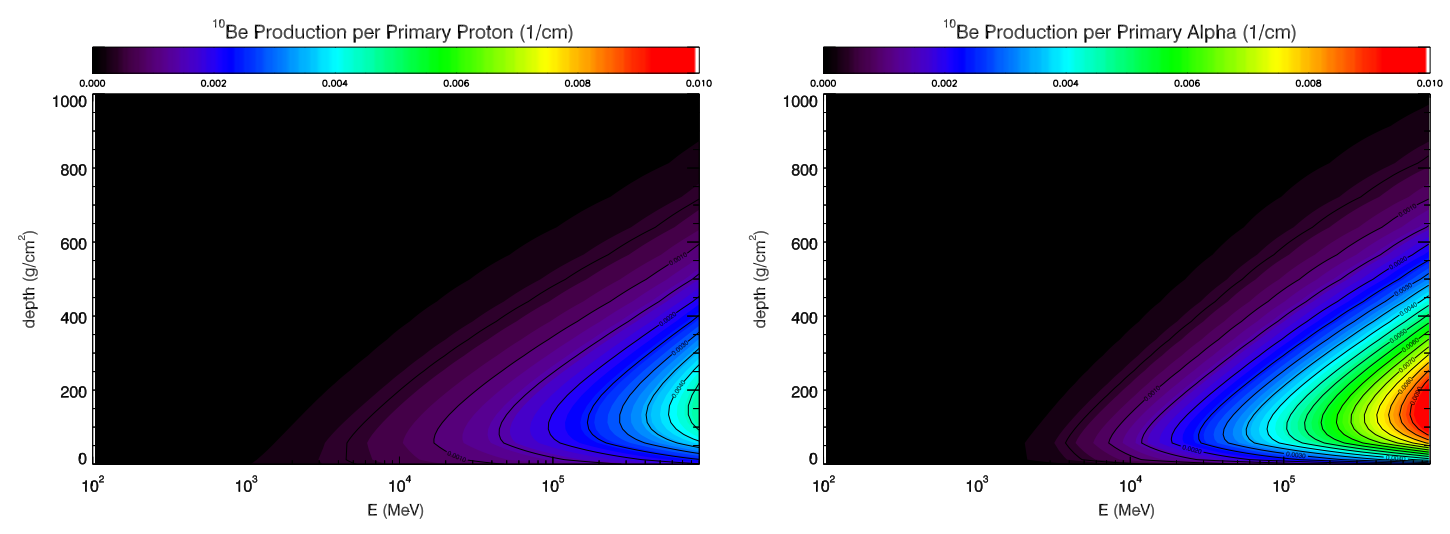

Figure 1: Production rate of ${ }^{10} \mathrm{Be}$ per primary protons (left panel) as well as primary alpha particles (right panel) with energies between $100 \mathrm{MeV}$ and $1 \mathrm{TeV}$. Computations have been performed for 30 different atmospheric depths between 0 and $1000 \mathrm{~g} / \mathrm{cm}^{2}$.

the world-wide Neutron Monitor network (see e.g. Tylka and Dietrich, 2009). The missing GLE events did not match the criteria for a spectral analysis and thus have been neglected in this study.

\section{Computation Method}

As discussed by Matthiä et al. (2013), the production $P$ of a cosmogenic radionuclide of type $j$ can be described by

$$
\begin{aligned}
P_{j}\left(E_{p}, x\right) & =\sum_{i} N_{i} \int_{E_{C}}^{\infty} \frac{d F_{p}}{d E_{p} d t} d E_{p} \sum_{k} \int_{0}^{\infty} \sigma_{i j k}\left(E_{k}\right) \cdot f\left(E_{p}, E_{k}, x\right) d E_{k} \\
& =\int_{E_{C}}^{\infty} \frac{d F_{p}}{d E_{p} d t} d E_{p} \cdot Y_{j}\left(E_{p}, x\right)
\end{aligned}
$$

a function of the differential primary particle fluence rate $d F_{p} /\left(d E_{p} d t\right)$, which gives the number of primary particles of species $p$ per area and time on top of the atmosphere (TOA) and the secondary particle fluence at a specific atmospheric depth $x, f\left(E_{p}, E_{k}, x\right)$ giving the number of secondary particles of type $k$ with an energy $E_{k}$ produced by a primary particle with an energy $E_{p}$. Furthermore, $N$ represents the density of the target atom of species $i$ while $\sigma_{i j k}$ represents the cross sections for the production of the cosmogenic radionuclide of type $j$. Thus, the yield function $Y_{j}\left(E_{p}, x\right)$ gives the number of cosmogenic radionuclides produced due to a single primary particle with the energy $E_{p}$ at a certain atmospheric depth $x$. In addition the primary particle intensity on TOA strongly depends on the geographic location, here represented by the cutoff energy $E_{C}$, i.e. the minimum energy a particle must have in order to be able to reach a certain location. The corresponding cutoff rigidity values $R_{C}$ thereby strongly depend on the magnetic field configuration and, thus, also vary on larger but also shorter time scales (see e.g. Herbst et al., 2013).

Using the GEANT4 Monte-Carlo software (version 4.9.1, see e.g. Agostinelli et al., 2003) as well as the PLANETOCOSMICS tool (Desorgher, 2006) the interactions of the primary particles with the surrounding atmospheric material were computed. The computations were performed in the energy range between $100 \mathrm{MeV}$ and $1 \mathrm{TeV}$, and energy range divided into 150 logarithmic equidistant energy bins. As pointed out by Matthiä et al. (2013) the contribution of primary particles with lower energies is negligible. Exemplarily, Fig. 1 shows the production rate of ${ }^{10} \mathrm{Be}$ per primary particle due to primary protons (left panel) as well as primary alpha particles (right panel). As can be seen, the higher the energy of the primary particle the 


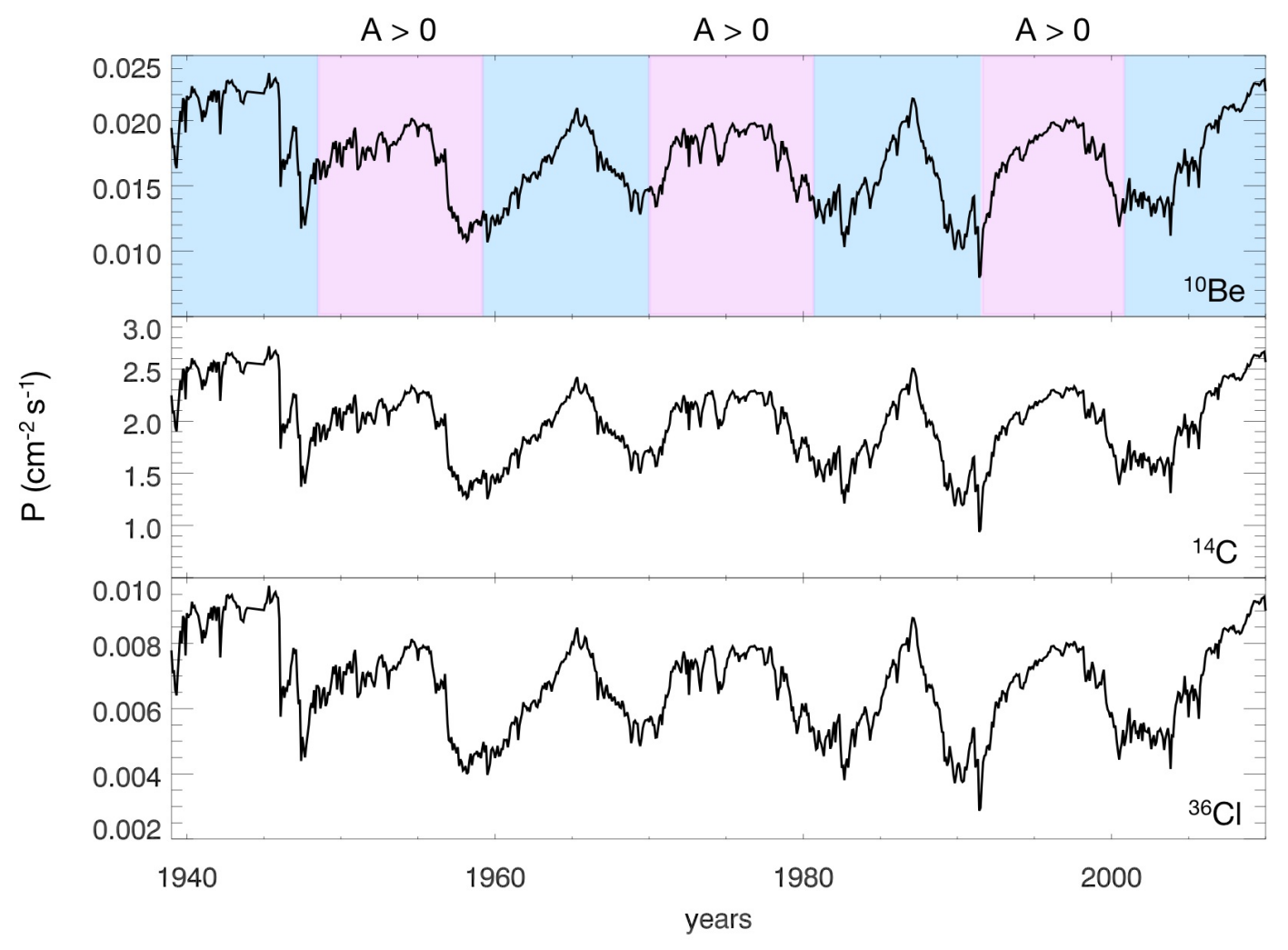

Figure 2: Computed global ${ }^{10} \mathrm{Be},{ }^{14} \mathrm{C}$ and ${ }^{36} \mathrm{Cl}$ production due to GCRs (monthly mean) over the past six solar cycles.

deeper it is able to penetrate into the atmosphere. Consequently, the ${ }^{10} \mathrm{Be}$ production per primary particle is increasing with increasing primary particle energy until a production maximum at around $150-200 \mathrm{~g} / \mathrm{cm}^{2}$ is reached. Moreover, Fig. 1 reveals that more ${ }^{10} \mathrm{Be}$ atoms per $\mathrm{cm}$ are produced by primary alpha particles than by primary protons.

\section{The production of ${ }^{10} \mathrm{Be},{ }^{14} \mathrm{C}$ and ${ }^{36} \mathrm{Cl}$ due to $\mathrm{GCRs}$}

In order to compute the production rates of the cosmogenic radionuclides ${ }^{10} \mathrm{Be},{ }^{14} \mathrm{C}$ and ${ }^{36} \mathrm{Cl}$ due to GCRs the yield function $Y_{j}\left(E_{p}, x\right)$ has to be multiplied with the modulated primary particle spectrum at 1 AU (see Eq. (1.1)) for which we use the proton and alpha particle LIS models by Usoskin et al. (2011a). Note, however, that the use of different LIS models has a strong influence on the production rate values (see e.g. Herbst et al., 2010).

Because GCRs continuously bombard the Earth the cosmogenic radionuclides produced by their interactions form a background production anti-correlated to the solar activity. Since the late 1930's continuous ground-based measurements of the GCR component are available, first with ionization chambers later with Neutron Monitors. Using those measurements Usoskin et al. (2011b) reconstructed the solar modulation parameter $\phi$ covering the last five solar cycles (1939 - today). By taking this reconstruction as well as the magnetic field variations into account we were able to reconstruct the global GCR-induced cosmogenic radionuclide production throughout this period. Figure 2 shows the computed global monthly-mean production rates of ${ }^{10} \mathrm{Be}$ (upper panel), ${ }^{14} \mathrm{C}$ (middle panel) as well as ${ }^{36} \mathrm{Cl}$ (lower panel). Because the three radionuclides are produced by similar physical processes the shape of their time dependent production is quite 


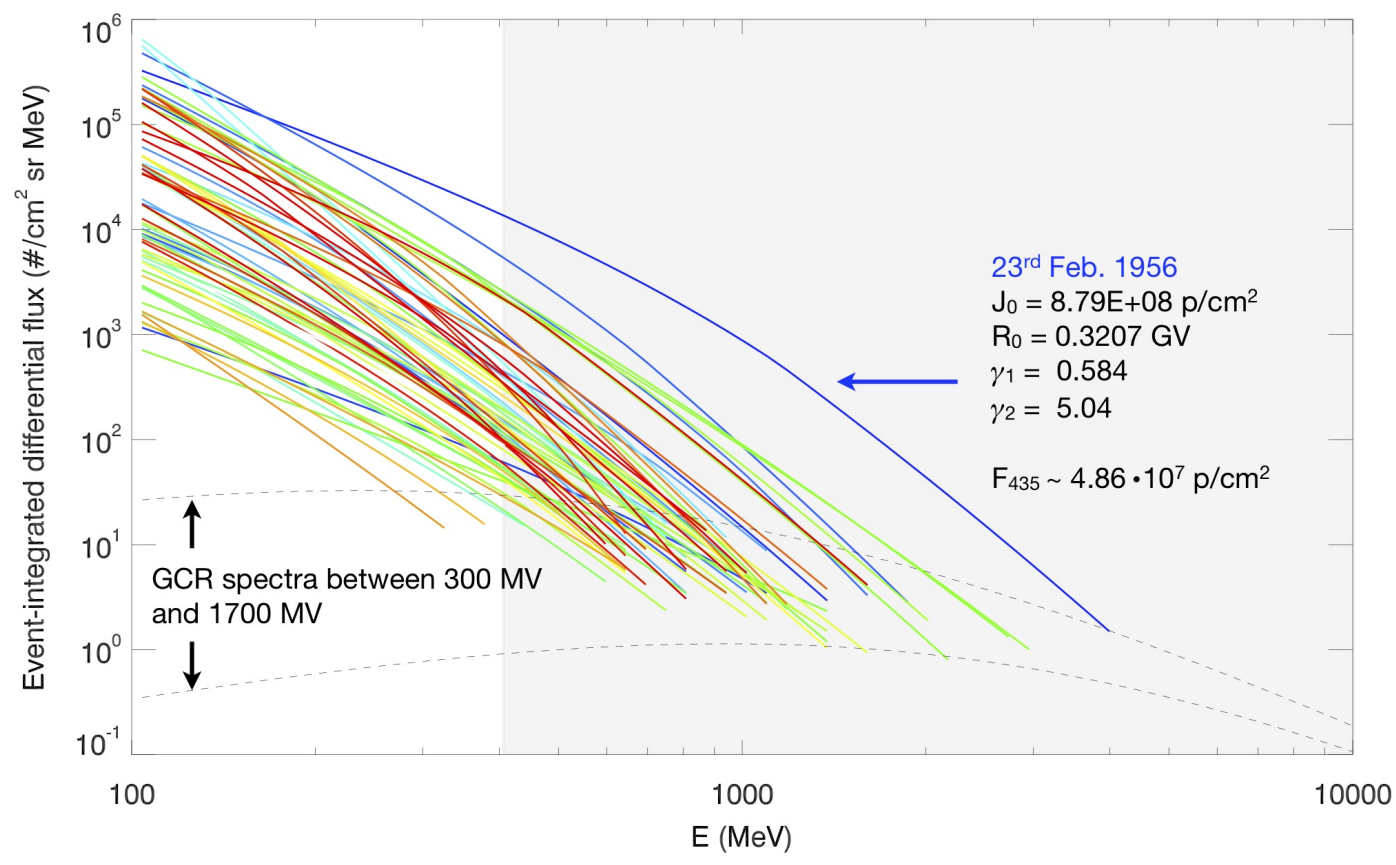

Figure 3: Energy spectra of 58 GLE events which occurred within the past five solar cycles.

similar. Thus, in all records the information of the 11- as well as 22-year solar cycle is visible. However, the global production rate values differ significantly from each other: ${ }^{14} \mathrm{C}$ is the most abundant radionuclide in the atmosphere while ${ }^{36} \mathrm{Cl}$ is about 20 times less abundant.

\section{Influence of modern GLE events on the production of ${ }^{10} \mathrm{Be},{ }^{14} \mathrm{C}$ and ${ }^{36} \mathrm{Cl}$}

Despite the GCR component the intensity of low energetic particles near the Earth can increase by orders of magnitude during strong SEP events. Of special interest for the production of the cosmogenic radionuclides are GLE events which can be detected by ground-basted instruments such as Neutron Monitors. Over the past five solar cycles 72 GLE events have been measured. Recently, Tylka and Dietrich (2009) investigated the energy spectra of the GLE events and were able to reconstruct the event-integrated fluxes in form of Band functions, describing the energy spectrum as an exponentially roll-over of two power-law spectra. The energy-dependent event-integrated differential fluxes of the GLE events are displayed in Fig. 3 (colored lines). In addition the GCR flux during solar minimum and solar maximum conditions are shown as dashed lines while the gray-shaded area marks the energy range important for the production of the cosmogenic radionuclides. Note that the strongest GLE event (GLE5) detected by now has occurred on February, $23^{\text {rd }} 1956$ (blue line, GLE 5). According to Kovaltsov et al. (2014) the event had an integrated flux above $200 \mathrm{MeV}$ of $F_{200}=1.2 \cdot 10^{8}$ protons per $\mathrm{cm}^{2}$, exceeding the second strongest event (GLE 10, 12 Nov. 1960) by almost a factor of two.

Using these proton spectra as input to Eq. 2.1, we now are also able to investigate the global production due to the modern GLE events. The results for the production of ${ }^{10} \mathrm{Be}$ are shown in Fig. 4. The upper panel displays the monthly-mean global ${ }^{10} \mathrm{Be}$ production rates due to the GCR-induced background (black line) as well as the GLE-induced production increases (red spikes). Furthermore, the gray and light blue shaded areas represent detection thresholds of two times the GCR background as well as the two sigma level, respectively. 

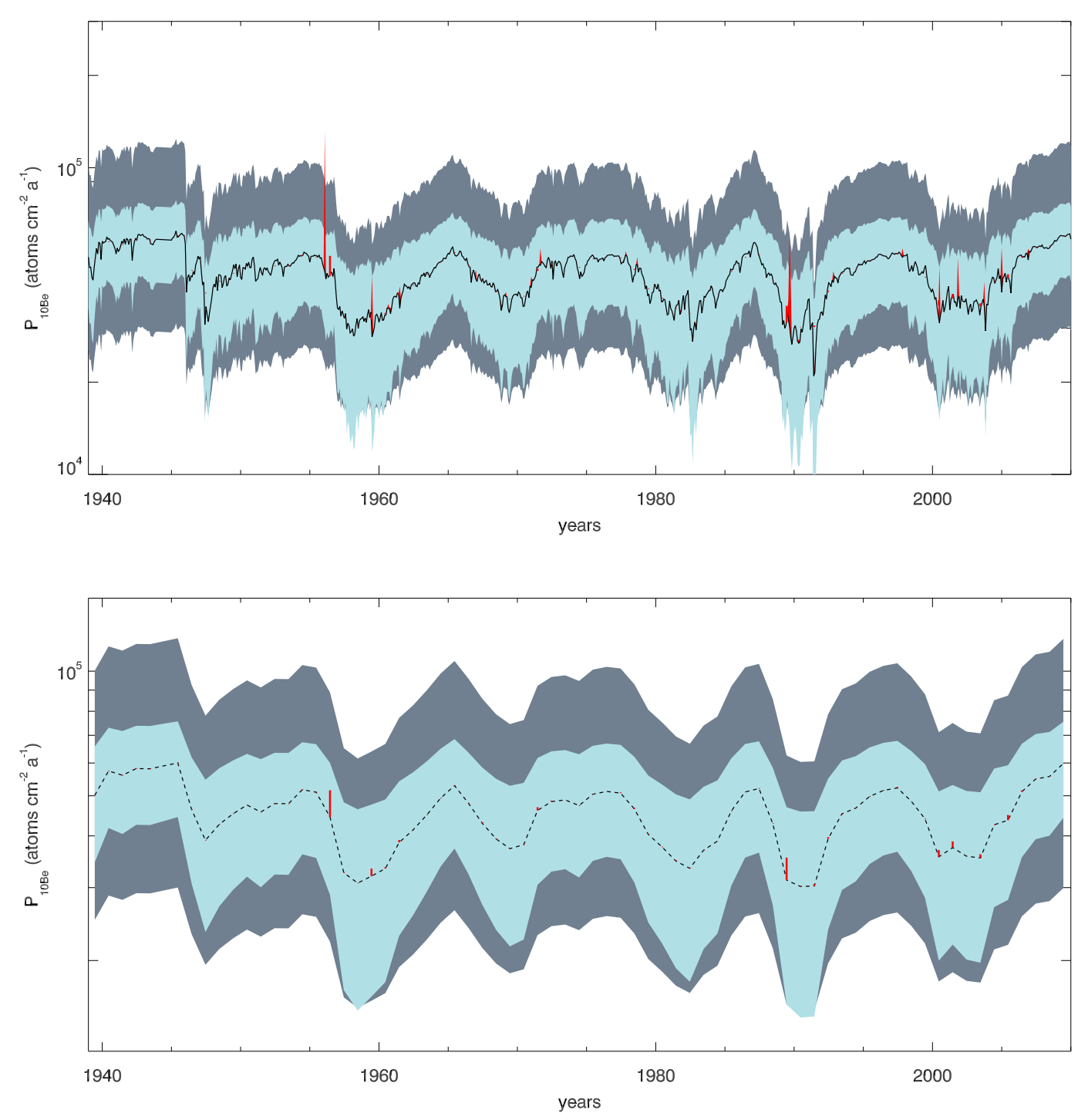

Figure 4: Energy spectra of 58 GLE events which occurred within the past five solar cycles.

As can be seen, in the computed monthly-mean production rate values GLE 5 exceeds both detection levels and would have caused an increase of about $25 \%$. Unfortunately, the measured radionuclide production rates from ice-sheets, tree rings or sediments have at best a yearly-mean averaged signal. Accordingly, the lower panel of Fig. 4 shows the computed yearly-mean averaged production rate values. From this study it becomes obvious that non of the investigated GLE events is strong enough to produce detectable signals in recent ${ }^{10} \mathrm{Be}$ records.

\section{Conclusions}

Immediately the question arises how strong the actual events should have had been in order to imprint a detectable increase above the two-times GCR background. The number of events needed to produce such a signal during the times the GLEs have occurred is shown in Fig. $5\left({ }^{10} \mathrm{Be}\right.$ in magenta, ${ }^{14} \mathrm{C}$ in black and ${ }^{36} \mathrm{Cl}$ 


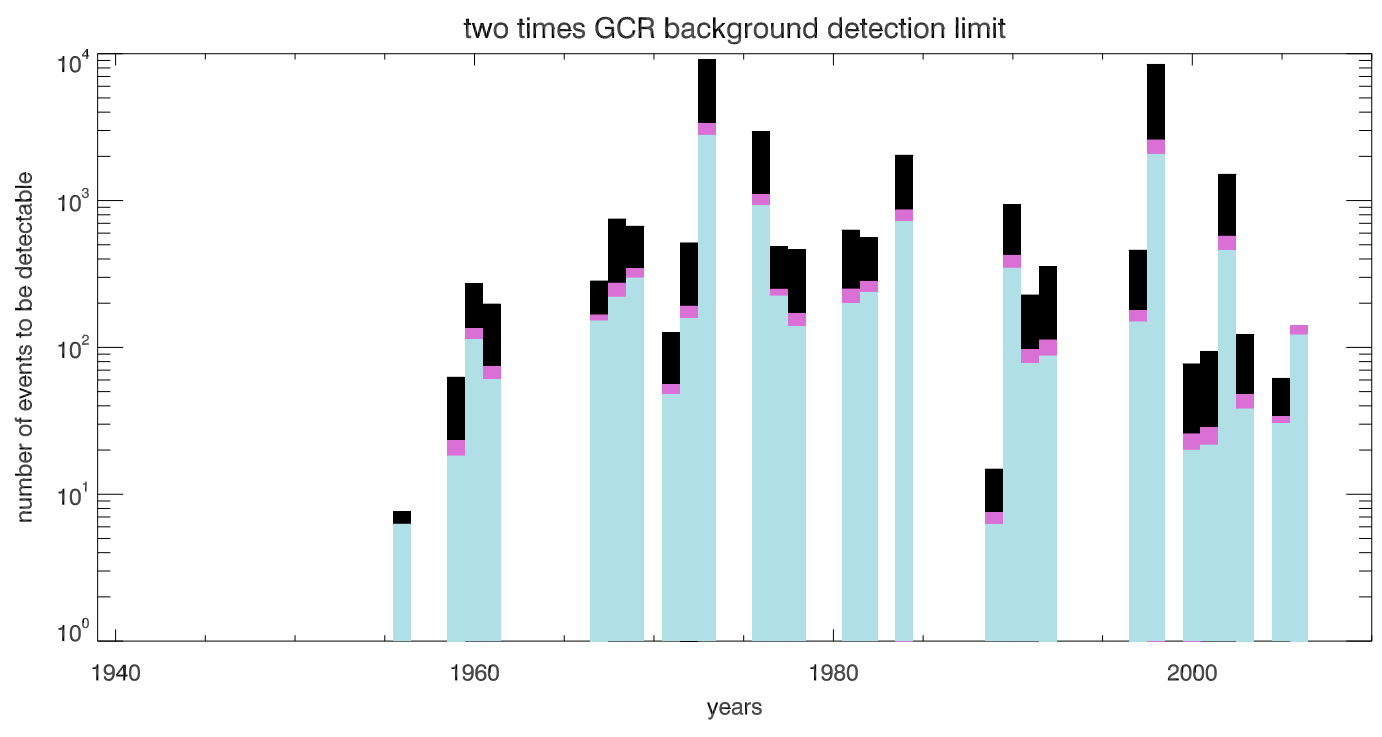

Figure 5: Number of events necessary which would have had to occur in order to produce a detectable signal in the ${ }^{10} \mathrm{Be}$ (magenta), ${ }^{14} \mathrm{C}$ (black) and ${ }^{36} \mathrm{Cl}$ (blue) records.

in blue). In case of GLE 5 a single event with a fluence of at least $F_{200}=8.4 \cdot 10^{8}$ protons per $\mathrm{cm}^{2}$ (or seven events in the order of GLE5 within the same year) would have had to occur in order to produce a detectable signal in e.g. the ${ }^{10} \mathrm{Be}$ records. However, in the majority of the GLEs fluences hundreds or thousands of times higher than the actual GLE fluence would have been mandatory. Moreover, this study reveals another important fact: the radionuclide most sensitive to almost all GLE events investigated is ${ }^{36} \mathrm{Cl}$ (blue) while the least sensitive one is ${ }^{14} \mathrm{C}$ (black).

\section{References}

S. Agostinelli, J. Allison, K. Amako et al. Geant4 - a simulation toolkit. Nuclear Instruments and Methods in Physics Research Section A, vol. 506, 250-303, 2003.

Caballero-Lopez, R. A. and H. Moraal. Limitations of the force field equation to describe cosmic ray modulation. J. Geophys. Res., 109, A12102, 2004.

Desorgher, L. The PLANETOCOSMICS code, Tech. rep., http://cosray.unibe.ch/laurent/planetocosmics, 2006.

Dunai, T. Cosmogenic Nuclides. Cambridge University Press, 2010.

Heikkilä, U., J. Beer and J. Feichter. Modeling cosmogenic radionuclides ${ }^{10}$ Be and ${ }^{7}$ Be during the Maunder Minimum using the ECHAM5-HAM General Circulation Model. Atmos. Chem. Phys., 8, 2797-2809, 2008.

Herbst, K., A. Kopp, B. Heber, F. Steinhilber, H. Fichtner, K. Scherer and D.Matthiä On the importance of the Local Interstellar Spectrum for the Solar Modulation Parameter. J. Geophys. Res., 115, D00I20, 2010.

Herbst, K., B. Heber, A. Kopp, O. Sternal and F. Steinhilber. The local interstellar spectrum beyond the heliopause: what can be learned from Voyager in the inner Heliosheath? Astrophys. J., 761, 17, 2012. 
Herbst, K., A. Kopp and B. Heber. Influence of the terrestrial magnetic field geometry on the cutoff rigidity of cosmic ray particles. Ann. Geophys., 31, 1637-1643, 2013.

Kovaltsov, G., I. G. Usoskin, E. W. Cliver, W. F. Dietrich and A. J. Tylka Fluence Ordering of Solar Energetic Proton Events Using Cosmogenic Radionuclide Data. Solar Phys, 289, 4691-4700, 2014.

Matthiä, D., K. Herbst, B. Heber, T. Berger and G. Reitz. ${ }^{10}$ Be Production in the Atmosphere by Galactic Cosmic Rays. Space Sci. Rev., 176, 333-342, 2013.

H. Moraal. Cosmic-Ray Modulation Equations. Space Sci. Rev., 291-311, 2011.

Mewaldt, R. A., M. D. Looper, C. M. S. Cohen, D. K. Haggerty, A. W. Labrador, R. A. Leske, G. M. Mason, J. E. Mazur and T. T. von Rosenvinge. Energy Spectra, Composition, and Other Properties of Ground-Level Events During Solar Cycle 23. Space Sci. Rev., 97-120, 2012.

Parker, E. N. The passage of energetic charged particles through interplanetary space. Planet. Space Sci., 13, 9-49, 1965.

Scherer, K., H. Fichtner, R. D. Strauss, S. E. S. Ferreira, M. S Potgieter and H. J. Fahr. On Cosmic Ray Modulation Beyond the Heliopause: Where is the Modulation Boundary?. Astrophys. J. , 735, 128-133, 2011.

Simpson, J. A. Elemental and isotopic composition of the galactic cosmic rays. Ann.Rev.Nucl.Part.Sci., 33, 323-381, 1983.

Simpson, J. A. The Cosmic Ray Nucleonic Component: The Invention and Scientific Uses of the Neutron Monitor. Space Sci. Rev., 93, 11-32, 2000.

Stoker, P. H. Relativistic Solar Proton Events. Space Sci. Rev., 73, 327-385, 1995.

Strauss, R. D., M. S. Potgieter, A. Kopp and I. Büsching. On the propagation times and energy losses of cosmic rays in the heliosphere. J. Geophys. Res., 116, 12105, 2011.

Tylka, A. J. and W. F. Dietrich. A new and comprehensive analysis of proton spectra in ground-level encahnced (GLE) solar particle events. In: 31th International Cosmic Ray Conference, Universal Academy Press, Lodz, 2009.

Usoskin, I. G., G. A. Brazlevskaia and G. A. Kovaltsov. Solar modulation parameter for cosmic rays since 1936 reconstructed from ground-based neutron monitors and ionization chambers. J. Geophys. Res., 116, A02104, 2011

Usoskin, I. G., G. A. Kovaltsov, I. A. Mironova, A. J. Tylka and W. F. Dietrich. Ionization effect of solar particle GLE events in low and middle atmosphere. Atm. Chem. and Phys., 11, 1979-1988, 2011 